\title{
Validating relativistic models of nuclear structure against theoretical, experimental, and observational constraints
}

\author{
J. Piekarewicz ${ }^{1}$ \\ ${ }^{1}$ Department of Physics, Florida State University, Tallahassee, FL 32306
}

(Dated: October 28, 2018)

\begin{abstract}
Relativistic mean-field models of nuclear structure have been enormously successful at reproducing ground-state properties of finite nuclei throughout the periodic table using a handful of accurately calibrated parameters. In this contribution we use powerful theoretical, experimental, and observational constraints - not employed in the calibration procedure - to validate two such models: NL3 and FSUGold. It is observed that FSUGold is consistent with all these constraints, except perhaps for a high density equation of state that appears mildly softer than required by astronomical observations. It is argued that incorporating such constrains goes a long way in removing much of the ambiguity left over from the standard calibrating procedure.
\end{abstract}

PACS numbers: 21.65.+f,26.60.+c,21.30.Fe 


\section{INTRODUCTION}

Mean field descriptions of the ground-state properties of medium to heavy nuclei have enjoyed enormous success. These highly economical descriptions encode a great amount of physics in a handful of model parameters that are calibrated to a few ground-state properties of a representative set of medium to heavy nuclei. An example of such a successful paradigm is the relativistic NL3 parameter set of Lalazissis, Ring, and collaborators [1, 2].

Yet by their mere nature, such mean-field models are untested away from their narrow window of applicability. Whereas models fitted to the ground-state properties of finite nuclei all tend to agree on the saturation properties of symmetric nuclear matter, they widely disagree on its density and isospin dependence [3, 4]. To resolve this ambiguity, an effort has been made to incorporate into the calibration procedure breathing-mode energies of heavy nuclei with neutron-proton asymmetries $[b \equiv(N-Z) / A]$ ranging from $b=0.11$ to $b=0.21[5,6]$. Although these approaches - combined with improved experimental data and analyzes - have helped narrow down the range of acceptable values of the incompressibility coefficient of symmetric nuclear matter to $K=230 \pm 10 \mathrm{MeV}$ [7, 8, 9], the density dependence of both symmetric-nuclear and pure-neutron matter remains largely undetermined. Thus, it is the aim of this contribution to test the validity of two accurately-calibrated relativistic mean-field models — NL3 [1, 2] and FSUGold [5] — against recent theoretical, experimental, and observational constraints not employed in the calibration procedure. In this manner we aim to establish the extent to which models that were accurately calibrated around nuclear saturation density may be reliably extrapolated to the low- and high-density regimes.

From the theoretical perspective, powerful arguments have provided critical insights into the behavior of pure neutron matter at low densities. Indeed, the low-density behavior of dilute Fermi gases with very large scattering lengths is universal, in that its energy equals that of the free Fermi gas up to a dimensionless universal constant of the order of 1/2 [10, 11]. Yet pure neutron matter deviates from unitarity due to its relatively large effective range. Fortunately, effective-range corrections to unitarity were recently computed by Schwenk and Pethick [12]. Such a model-independent approach will be used to test the validity of meanfield models away from their region of applicability. Moreover, it will be shown how such powerful theoretical constraints may be used to rule out a variety of accurately calibrated models [3, 4] .

Laboratory experiments with heavy ions have played a critical role in constraining the nuclear equation of state. By tuning the energy of the colliding beams and the neutron-proton asymmetry, heavy-ion collisions probe vast regions of the phase diagram. For example, by compressing nuclear matter to pressures never before attained under laboratory conditions, the equation of state of symmetric nuclear matter was determined up to densities of about 4-to-5 times that of normal nuclear matter [13]. Moreover, low-density constraints on the equation of state of neutron-rich matter are starting to emerge from the distribution of fragments in medium-energy collisions. In particular, by plotting the data in an ingenious manner, a powerful scaling relation - known as isoscaling [14, 15] — was uncovered and shown to be sensitive to the low-density behavior of the symmetry energy [16]. Note that the symmetry energy equals to an excellent approximation the difference between the energy of pure neutron matter and that of symmetric matter.

Finally, enormous advances in both land- and spaced-based observatories have brought the fields of nuclear physics and astrophysics closer than ever before. In the particular case of

neutron star structure and its intimate connection to the equation of state of dense matter, a 
few recent developments are worth mentioning. Among these, observations of neutron-starwhite-dwarf binaries with the Arecibo radio telescope have resulted in the largest neutronstar mass ever reported $M(\mathrm{PSR} J 0751+1807)=2.1 \pm 0.2 M_{\odot}$ [17]. The limiting mass of a neutron star represents the optimal (and perhaps unique) way of constraining the high density component of the equation of state. If the above error bars can be narrowed down any further, a significant number of (soft) models of the equation of state will be ruled out [18, 19]. Moreover, transient phenomena - such as X-ray bursts powered by the nuclear burning of $\mathrm{H} / \mathrm{He}$ in low-mass X-ray binaries — provide a powerful observational constraint on the long-sought mass-vs-radius relationship of a neutron star. For example, the massto-radius ratio of the neutron star in the low-mass X-ray binary EXO 0748-676 has been recently constrained by detecting the gravitational redshift of certain absorption lines [20]. More recently, by combining this result with additional observational constraints, both the mass and radius of the neutron star in EXO 0748-676 were individually determined to be $M \geq 2.10 \pm 0.28 M_{\odot}$ and $R \geq 13.8 \pm 1.80 \mathrm{~km}[21]$ — an interpretation that appears to rule out all soft equations of state. For a comprehensive recent account on constraints on the equation of state from neutron-star observations see Ref. [18].

The manuscript has been organized as follows. In Sec. II a brief review of the relativistic formalism will be provided. Particular emphasis will be placed on those model parameters that are loosely constrained by existent ground-state observables and the extent to which they may be firmly determined by the various constraints discussed above. Next, in Sec. III] results will be presented for a variety of low- and high-density observables that will be compared against available theoretical, experimental, and observational results. Finally, we offer a summary and conclusions in Sec. IV.

\section{FORMALISM}

The Lagrangian density employed in this work is rooted on the seminal work of Walecka, Serot, and their many collaborators (see Refs. [22, 23, 24] and references therein). Since first published by Walecka more than three decades ago [24], several refinements have been implemented to improve the quantitative standing of the model. In the present work we employ an interacting Lagrangian density of the following form [5, 25, 26] :

$$
\begin{aligned}
\mathscr{L}_{\text {int }} & =\bar{\psi}\left[g_{\mathrm{s}} \phi-\left(g_{\mathrm{v}} V_{\mu}+\frac{g_{\rho}}{2} \tau \cdot \mathbf{b}_{\mu}+\frac{e}{2}\left(1+\tau_{3}\right) A_{\mu}\right) \gamma^{\mu}\right] \psi \\
& -\frac{\kappa}{3 !}\left(g_{\mathrm{s}} \phi\right)^{3}-\frac{\lambda}{4 !}\left(g_{\mathrm{s}} \phi\right)^{4}+\frac{\zeta}{4 !}\left(g_{\mathrm{v}}^{2} V_{\mu} V^{\mu}\right)^{2}+\Lambda_{\mathrm{v}}\left(g_{\rho}^{2} \mathbf{b}_{\mu} \cdot \mathbf{b}^{\mu}\right)\left(g_{\mathrm{v}}^{2} V_{\mu} V^{\mu}\right) .
\end{aligned}
$$

The original Lagrangian density of Walecka consisted of an isodoublet nucleon field $(\psi)$ together with neutral scalar $(\phi)$ and vector $\left(V^{\mu}\right)$ fields coupled to the scalar density $(\bar{\psi} \psi)$ and conserved nucleon current $\left(\bar{\psi} \gamma^{\mu} \psi\right)$, respectively [24]. In spite of its simplicity (the model contains only two dimensionless coupling constants), symmetric nuclear matter saturates even when the model was solved at the mean-field level [24]. By adding additional contributions from a single isovector meson $\left(b^{\mu}\right)$ and the photon $\left(A^{\mu}\right)$, Horowitz and Serot [27] obtained results for the ground-state properties of finite nuclei that rivaled some of the most sophisticated non-relativistic calculations of the time. However, whereas the two dimensionless parameters in the original Walecka model could be adjusted to reproduce the nuclear saturation point, the incompressibility coefficient (now a prediction of the model) was too 


\begin{tabular}{|l||c|c|c|c|c|c|c|c|}
\hline Model & $m_{\mathrm{s}}$ & $g_{\mathrm{s}}^{2}$ & $g_{\mathrm{v}}^{2}$ & $g_{\rho}^{2}$ & $\kappa$ & $\lambda$ & $\zeta$ & $\Lambda_{\mathrm{v}}$ \\
\hline \hline NL3 & 508.1940 & 104.3871 & 165.5854 & 79.6000 & 3.8599 & -0.0159 & 0.0000 & 0.0000 \\
\hline FSUGold & 491.5000 & 112.1996 & 204.5469 & 138.4701 & 1.4203 & +0.0238 & 0.0600 & 0.0300 \\
\hline
\end{tabular}

TABLE I: Model parameters used in the calculations. The parameter $\kappa$ and the inverse scalar range $m_{\mathrm{s}}$ are given in $\mathrm{MeV}$. The nucleon, omega, and rho masses are kept fixed at $M=939 \mathrm{MeV}$, $m_{\omega}=782.5 \mathrm{MeV}$, and $m_{\rho}=763 \mathrm{MeV}$, respectively.

large $(K \gtrsim 500 \mathrm{MeV})$ as compared with existing data on breathing-mode energies [28]. To overcome this problem, Boguta and Bodmer introduced cubic $(\kappa)$ and quartic $(\lambda)$ scalar meson self-interactions that accounted for a significant softening of the equation of state $(K=150 \pm 50 \mathrm{MeV})$ [29]. Two parameters of the Lagrangian density of Eq. (1) remain to be discussed, namely, $\zeta$ and $\Lambda_{\mathrm{v}}$. Both of these parameters are set to zero in the enormously successful NL3 model, suggesting that the experimental data used in the calibration procedure is insensitive to the physics encoded in these parameters. Indeed, Müller and Serot found possible to build models with different values of $\zeta$ that reproduce the same observed properties at normal nuclear densities, but which yield maximum neutron star masses that differ by almost one solar mass [25]. This result indicates that observations of massive neutron stars - rather than laboratory experiments - may provide the only meaningful constraint on the high-density component of the equation of state. Finally, the isoscalar-isovector coupling constant $\Lambda_{\mathrm{v}}$ was added in Ref. [26] to modify the density dependence of the symmetry energy. It was found that models with different values of $\Lambda_{\mathrm{v}}$ reproduce the same exact properties of symmetric nuclear matter, but yield vastly different values for the neutron skin thickness of heavy nuclei and for the radii of neutron stars [30]. The Parity Radius Experiment (PREX) at the Jefferson Laboratory promises to measure the skin thickness of ${ }^{208} \mathrm{~Pb}$ accurately and model independently via parity-violating electron scattering [31, 32]. PREX will provide a unique experimental constraint on the density dependence of the symmetry energy due its strong correlation to the neutron skin of heavy nuclei [3].

\section{RESULTS}

In this section we compare the predictions from NL3 [1, 2] and FSUGold [5] against the theoretical, experimental, and observational constraints discussed in the Introduction. The effective parameters of the two models are listed in Table $\Pi$ and their predictions for several bulk properties of nuclear matter are tabulated in Table II. Note that $\rho_{0}, \varepsilon_{0}$, and $K$ denote the density, the binding energy per nucleon and incompressibility coefficient of symmetric nuclear matter while $J$ and $L$ denote the value and slope of the symmetry energy, all at saturation density. In particular, the pressure of pure neutron matter at saturation density is related to $L$ through the following expression:

$$
P_{\mathrm{PNM}}\left(\rho_{0}\right)=\frac{1}{3} \rho_{0} L .
$$




\begin{tabular}{|l||c|c|c|c|c|}
\hline Model & $\rho_{0}\left(\mathrm{fm}^{-3}\right)$ & $\varepsilon_{0}(\mathrm{MeV})$ & $K(\mathrm{MeV})$ & $J(\mathrm{MeV})$ & $L(\mathrm{MeV})$ \\
\hline \hline NL3 & 0.148 & -16.24 & 271 & 37.3 & 118.4 \\
FSUGold & 0.148 & -16.30 & 230 & 32.6 & 60.5 \\
\hline
\end{tabular}

TABLE II: Bulk parameters characterizing the energy of symmetric nuclear matter $\left(\rho_{0}, \varepsilon_{0}\right.$, and $K)$ and the symmetry energy $(J$ and $L)$ at saturation density.

\section{A. Theoretical Constraints}

Perhaps surprisingly, one of the most stringent constraints on the equation of state of low density neutron-rich matter emerges from theoretical considerations, namely, from the universality of dilute Fermi gases with an "infinite" scattering length $(a)$. In this limit the only energy scale in the problem is the Fermi energy $\left(\varepsilon_{\mathrm{F}}\right)$, so the energy per particle is constrained to be that of the free Fermi gas up to a dimensionless universal constant $(\xi)$ that is independent of the details of the two-body interaction [10]. That is,

$$
\frac{E}{N}=\xi \frac{3}{5} \varepsilon_{\mathrm{F}} .
$$

To date, the best theoretical estimates place the value of the universal constant around $\xi \approx 0.4[10,11,33,34]$.

While the neutron-neutron scattering length is large indeed $\left(a_{\mathrm{nn}}=-18.5 \mathrm{fm}\right)$, pure neutron matter deviates from unitarity due to a non-negligible value of the effective range of the neutron-neutron interaction $\left(r_{\mathrm{e}}=+2.7 \mathrm{fm}\right)$. Thus, corrections to the low-density equation of state of pure neutron matter must be computed for $k_{\mathrm{F}} \sim r_{\mathrm{e}}^{-1} \simeq 0.4 \mathrm{fm}^{-1}$. Such corrections have been recently computed by Schwenk and Pethick [12], with their results displayed as the red region in Fig 1. Also shown are the predictions of two microscopic models based on realistic two-body interactions, one of them being the venerated equation of state of Friedman and Pandharipande [35]. Finally, the predictions of NL3 and FSUGold are also shown. It is gratifying that the softening of the symmetry energy of FSUGold - caused by incorporating constraints from breathing-mode energies [5] — appears consistent with the physics of resonant Fermi gases. Such a powerful universal constraint should be routinely and explicitly incorporated into future determinations of density functionals. Indeed, such a constrain appears to rule out many of the models displayed in Fig. 2 of Ref. [3] .

\section{B. Experimental Constraints}

Laboratory experiments place important constraints on the equation of state of hadronic matter. Indeed, a variety of ground-state properties (primarily masses and charge radii) of finite nuclei are routinely incorporated into the calibration procedure of the models. However, the impact of heavy-ion experiments on these models is just starting to emerge. A particularly relevant example involves the distribution of fragments in medium-energy heavy ion collisions. It has been shown that the ratio of isotopic yields $\left[R_{21}(N, Z)\right]$ obeys a powerful scaling relation [14, 15] that is sensitive to the low-density behavior of the symmetry energy [16]. It is observed, quite naturally, that the reaction with neutron-rich nuclei (e.g., ${ }^{124} \mathrm{Sn}+{ }^{124} \mathrm{Sn}$ ) produces more neutron-rich and less proton-rich fragments relative to the neutron-deficient reaction (e.g., $\left.{ }^{112} \mathrm{Sn}+{ }^{112} \mathrm{Sn}\right)$. This makes the reaction yields particularly sensitive to the density dependence of the symmetry energy. For example, a stiff 


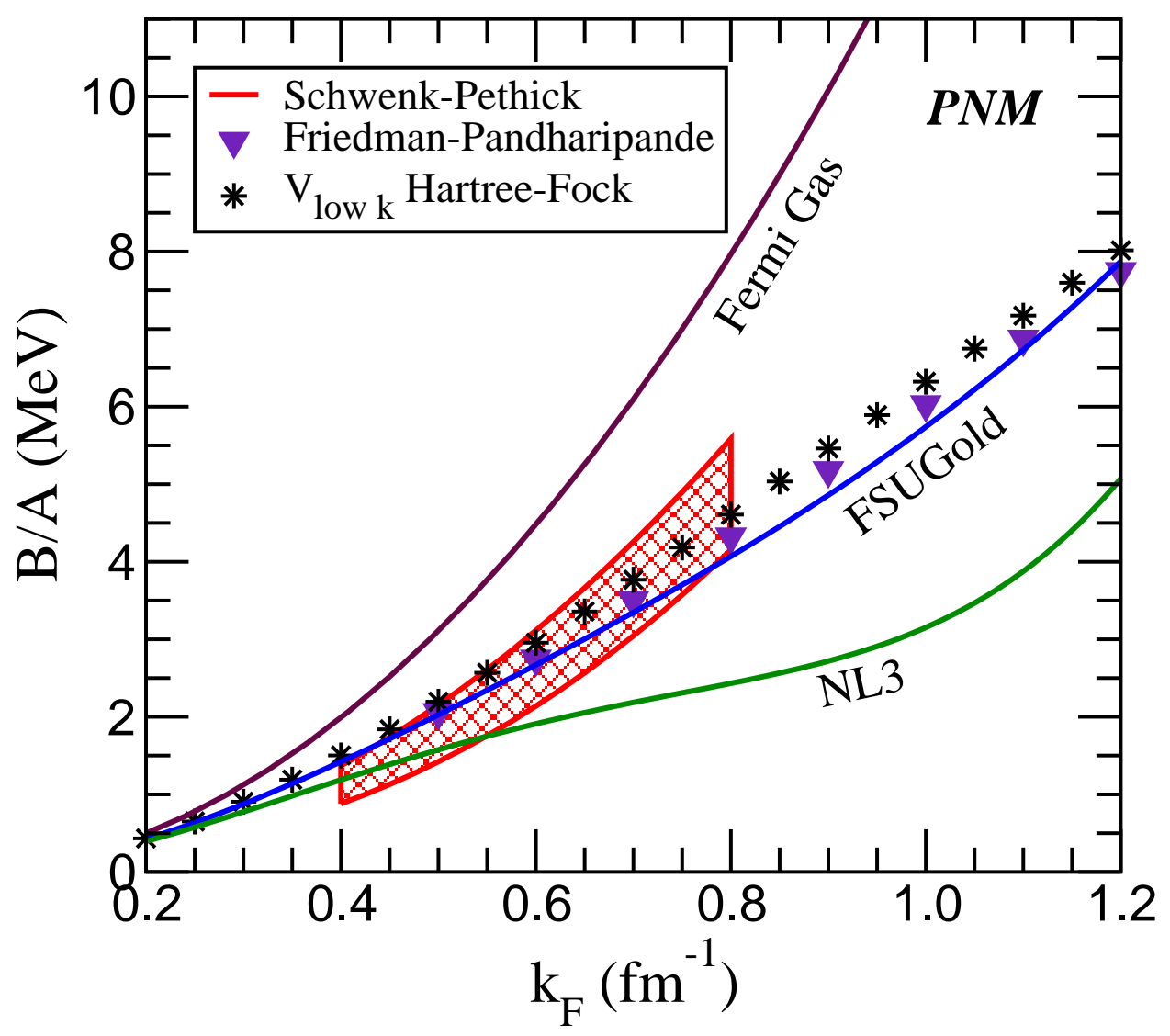

FIG. 1: (color online) Equation of state of pure neutron matter as a function of the Fermi momentum. Predictions are shown for the accurately calibrated NL3 [1, 2] (green line) and FSUGold [5] (blue line) parameter sets. Shown also are various microscopic descriptions - including a modelindependent result based on the physics of resonant Fermi gases by Schwenk and Pethick [12] (red region).

symmetry energy (such as the one displayed by NL3 in Fig. (2) imposes a stiff penalty on the system at high density for departing from the symmetric $(N=Z)$ limit. It is, however, the softer symmetry energy (such as FSUGold in Fig. 2) that imposes the stiffer penalty at the low densities relevant to the multifragmentation process. As such, one expects more neutron-rich fragments to be produced by a stiff rather than by a soft equation of state [36]. The experimental signature of this behavior is imprinted in a parameter than controls the variation of $R_{21}(N, Z)$ with $N$ for fixed $Z$ (a parameter usually denoted by $\alpha$ ). Using general thermodynamic arguments, the value of $\alpha$ was related to the change of the neutron chemical potential with the neutron-proton asymmetry [14, 15]. Note that it was precisely the study of the neutron chemical potential of neutron-rich nuclei at zero temperature (i.e., the neutron Fermi energy) that lead to the conclusion that models with a soft symmetry energy reach the neutron-drip line before those with a stiffer symmetry energy [37].

In a recent study, Shetty, Yennello, and Souliotis [38] used the scaling behavior of the fragment yields - coupled to a molecular dynamics simulation - to extract the density 


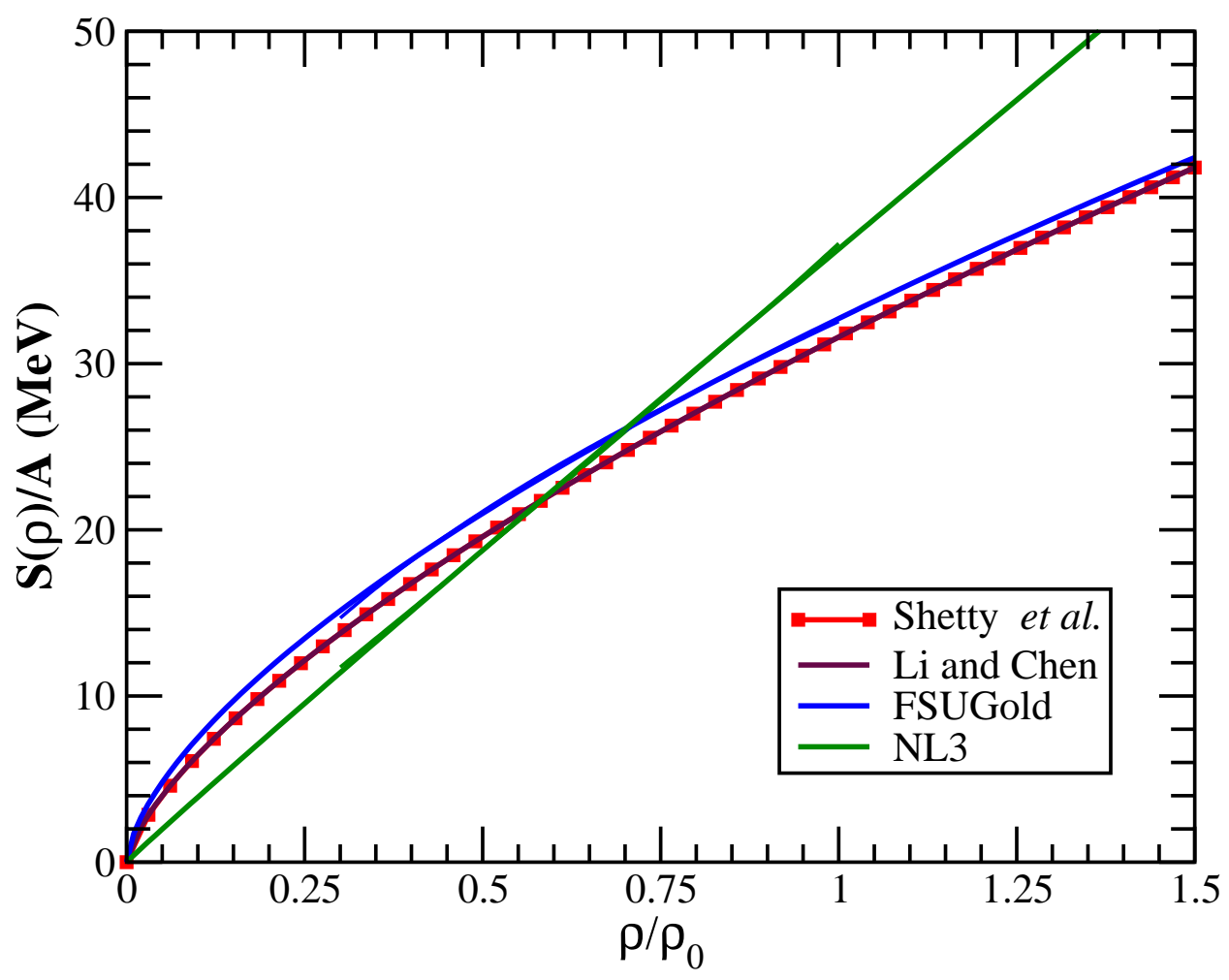

FIG. 2: (color online) Symmetry energy as a function of the baryon density (expressed in units of the saturation density $\rho_{0}=0.148 \mathrm{fm}^{-3}$ ). Predictions are shown from the NL3 [1, 2] (green line) and FSUGold [5] (blue line) models. Shown also are the results from Li and Chen [41] (maroon line) and the experimental analysis of Shetty and collaborators [38] (red symbols).

dependence of the symmetry energy. In the low density regime probed in the collisions, the density dependence of the symmetry energy may be parametrized according to the following simple formula:

$$
S(\rho)=S\left(\rho_{0}\right)\left(\frac{\rho}{\rho_{0}}\right)^{\gamma}
$$

To make contact with this approach, our theoretical results were fitted to the above formula in the $\rho=(0.3-1.0) \rho_{0}$ range, yielding values for the symmetry energy at saturation $\left[S\left(\rho_{0}\right)\right]$ and for the exponent $(\gamma)$ as displayed in Eq. (5). This same information is depicted in graphical form in Fig. 2. As in the case of pure neutron matter (see Fig. 1) it appears that the density dependence of the symmetry energy predicted by FSUGold - at least at low densities - is consistent with this experimental analysis. However, the experimental determination is not without controversy [39, 40]. Moreover, one should be aware that the connection between the collision of heavy ions and the zero-temperature equation of state 
is model dependent.

$$
S\left(\rho_{0}\right)=\left\{\begin{array}{ll}
31.6 \mathrm{MeV}, & \text { Ref. [38], } \\
31.6 \mathrm{MeV}, & \text { Ref. [41], } \\
32.7 \mathrm{MeV}, & \text { FSUGold, } \\
36.9 \mathrm{MeV}, & \text { NL3; }
\end{array} \quad \gamma= \begin{cases}0.69, & \text { Ref. [38] } \\
0.69, & \text { Ref. [41] } \\
0.64, & \text { FSUGold } \\
0.98, & \text { NL3. }\end{cases}\right.
$$

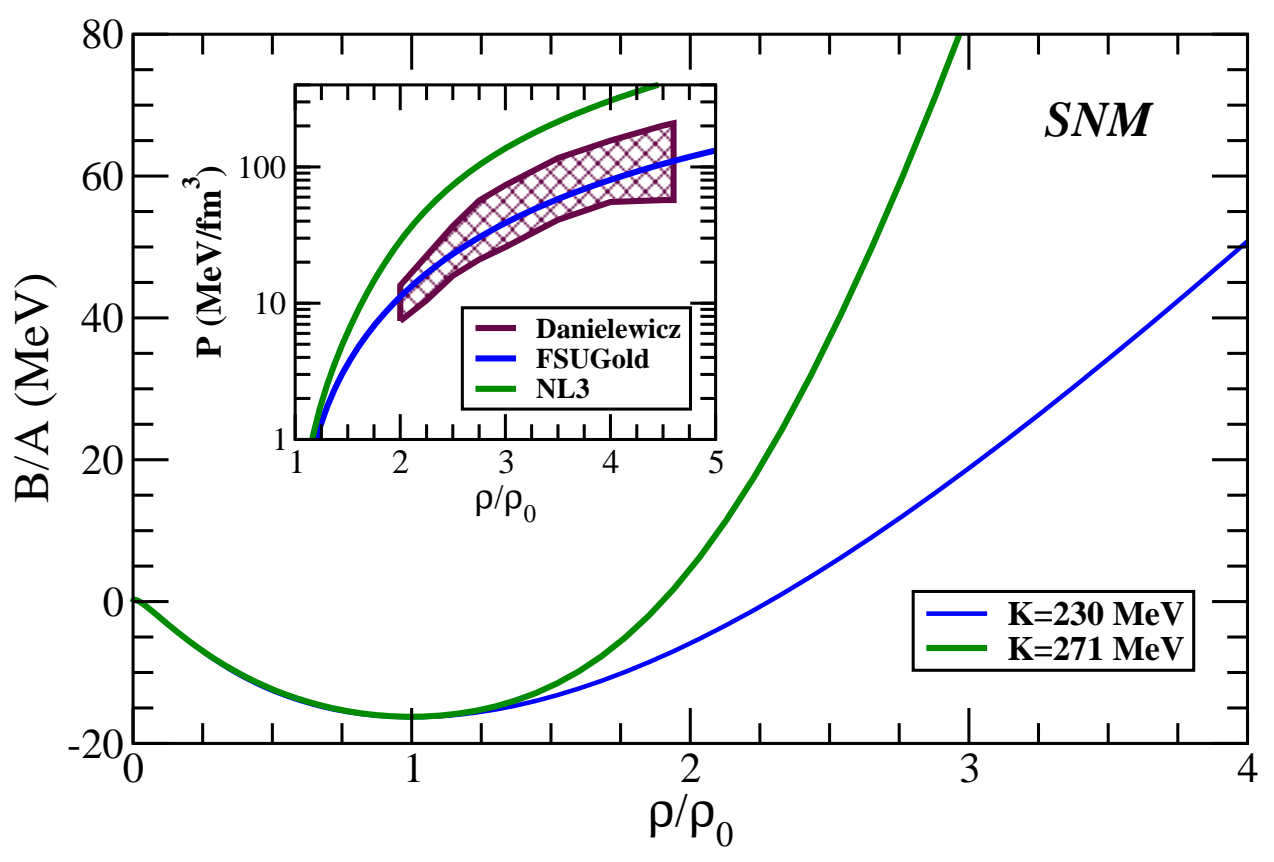

FIG. 3: (color online) Binding energy per nucleon as a function of baryon density (expressed in units of the saturation density $\rho_{0}=0.148 \mathrm{fm}^{-3}$ ) for symmetric nuclear matter. Theoretical predictions are shown for the NL3 [1, 2] (green line) and FSUGold [5] (blue line) models. Shown in the inset is a comparison between the equation of state extracted from energetic nuclear collisions [13] and the predictions of these two models.

Nuclear collisions may also be used to constrain the high-density behavior of nucleonic matter. To illustrate this point we display in Fig. 3 the binding energy per nucleon of symmetric nuclear matter as a function of the baryon density as predicted by both the NL3 and FSUGold models. Note that both models reproduce the equilibrium properties of symmetric nuclear matter and display the same quantitative behavior at densities below the saturation point. Yet their high-density predictions are significantly different. This emerges from a combination of two factors. First, FSUGold predicts an incompressibility coefficient $K$ considerably lower than NL3, namely, $230 \mathrm{MeV}$ vs $271 \mathrm{MeV}$ (see Table III). Second, and more importantly, FSUGold includes a self-energy coupling [denoted by $\zeta$ in Eq. (1)] that is responsible for a significant softening at high density. Note that the mixed isoscalarisovector coupling $\left(\Lambda_{\mathrm{v}}\right)$ plays no role in symmetric nuclear matter. We now compare the predictions of these two models against results obtained from energetic nuclear collisions that can compress baryonic matter to densities as high as those predicted to exist in the 
core of neutron stars. The inset in Fig. 3 provides us with such a comparison. By analyzing the manner in which matter flows after the collision of two energetic gold nuclei, the equation of state of symmetric nuclear matter was extracted up to densities of 4-to-5 times saturation density [13]. Figure 3 seems to rule out overly stiff equations of state (such as NL3). And while it continues to be gratifying that FSUGold is consistent with this analysis, one should reiterate that the connection between energetic nuclear collisions and the equation of state of cold nuclear matter is model dependent. Yet within these limitations, the same analysis [13] has been used to impose constraints on the equation of state of pure neutron matter by assuming two models — one soft and one stiff — for the unknown density dependence of the symmetry energy. The resulting equations of state are displayed in Fig. 4 alongside the predictions of both theoretical models. The FSUGold parametrization appears consistent with a relatively soft symmetry energy.

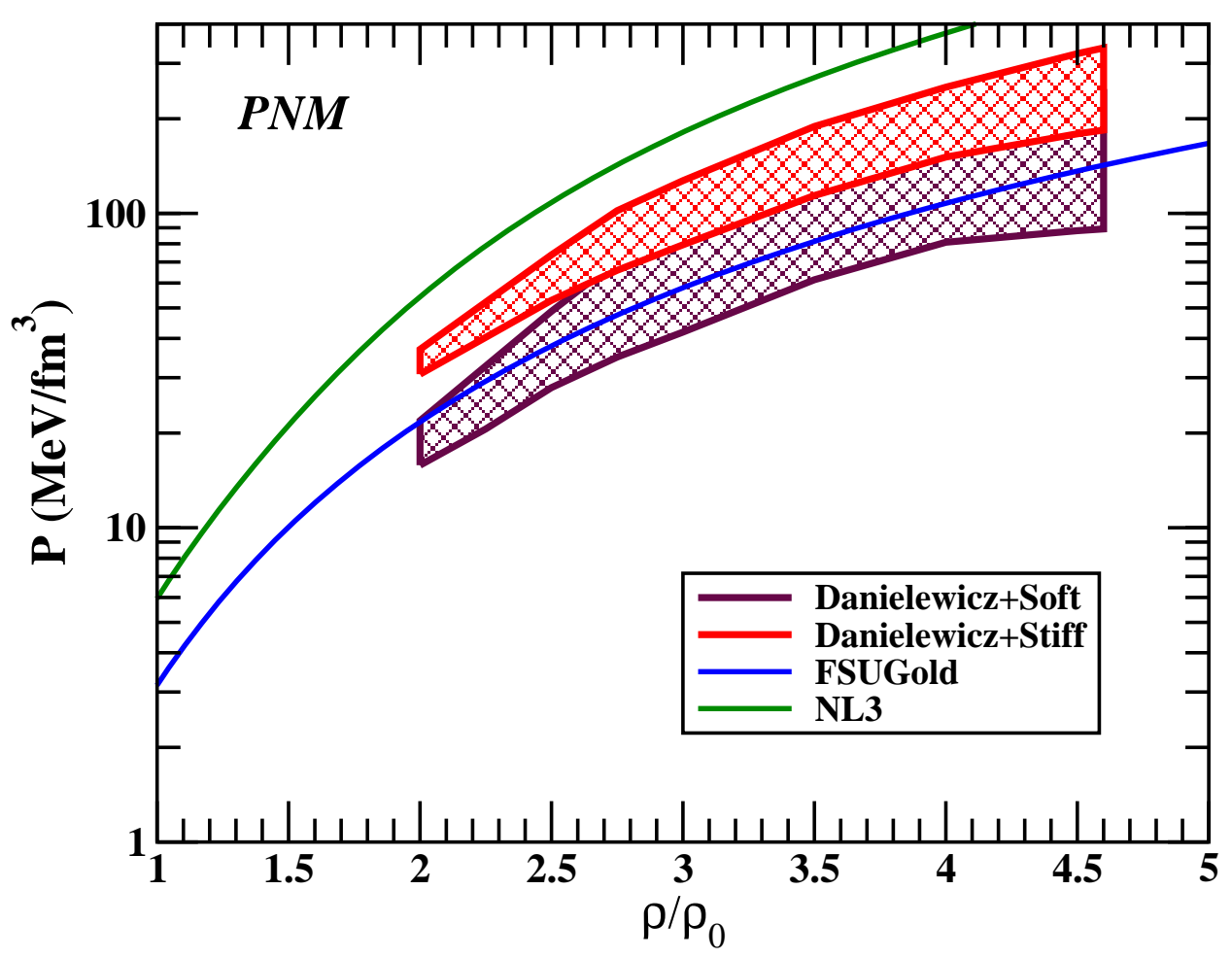

FIG. 4: (color online) The pressure as a function of baryon density (expressed in units of the saturation density $\rho_{0}=0.148 \mathrm{fm}^{-3}$ ) for pure neutron matter as extracted from energetic nuclear collisions [13] by assuming soft (maroon) and stiff (red) symmetry energies. Also shown are the theoretical predictions from the NL3 [1, 2] (green line) and FSUGold [5] (blue line) models.

\section{Observational Constraints}

Ultimately, the most reliable constraints on the high-density component of the equation of state will come from astronomical observations. Indeed, important constraints are starting 
to emerge from the combination of a large number of observations [18]. Here we limit ourselves to only two of them for their significant impact on the present analysis. For two other studies similar in spirit to the present one see the very recent references [42] and [43].

The first observation that impacts significantly on the present work is the one by Nice and collaborators at the Arecibo radio telescope [17]. Such observation of a neutron-star-whitedwarf binary system appears to suggest a neutron-star mass of $M($ PSR J0751+1807) $=$ $2.1 \pm 0.2 M_{\odot}$ (this is denoted by the red region in Figure 5). This appears to be the largest neutron-star mass ever reported and one that is significantly larger than those most accurately determined from double neutron-star binaries that display a mean of only about 1.35-1.40 $M_{\odot}$ with a very small dispersion. If the limits on the mass of PSR J0751+1807 can be tighten any further (after all, at the $2 \sigma$ level the observation accommodates the rather wide range of 1.6-2.5 $M_{\odot}$ ) it would practically pin down the high-density component of the equation of state. Indeed, in the particular case of the FSUGold model with a prediction of only $1.72 M_{\odot}$ for the limiting mass, this observation would demand a mild hardening of the equation of state at high densities. As first observed by Müller and Serot [25], in relativistic mean-field models this may be efficiently achieved by simply tuning the non-linear coupling $\zeta$, while leaving intact all other parameters of the model.

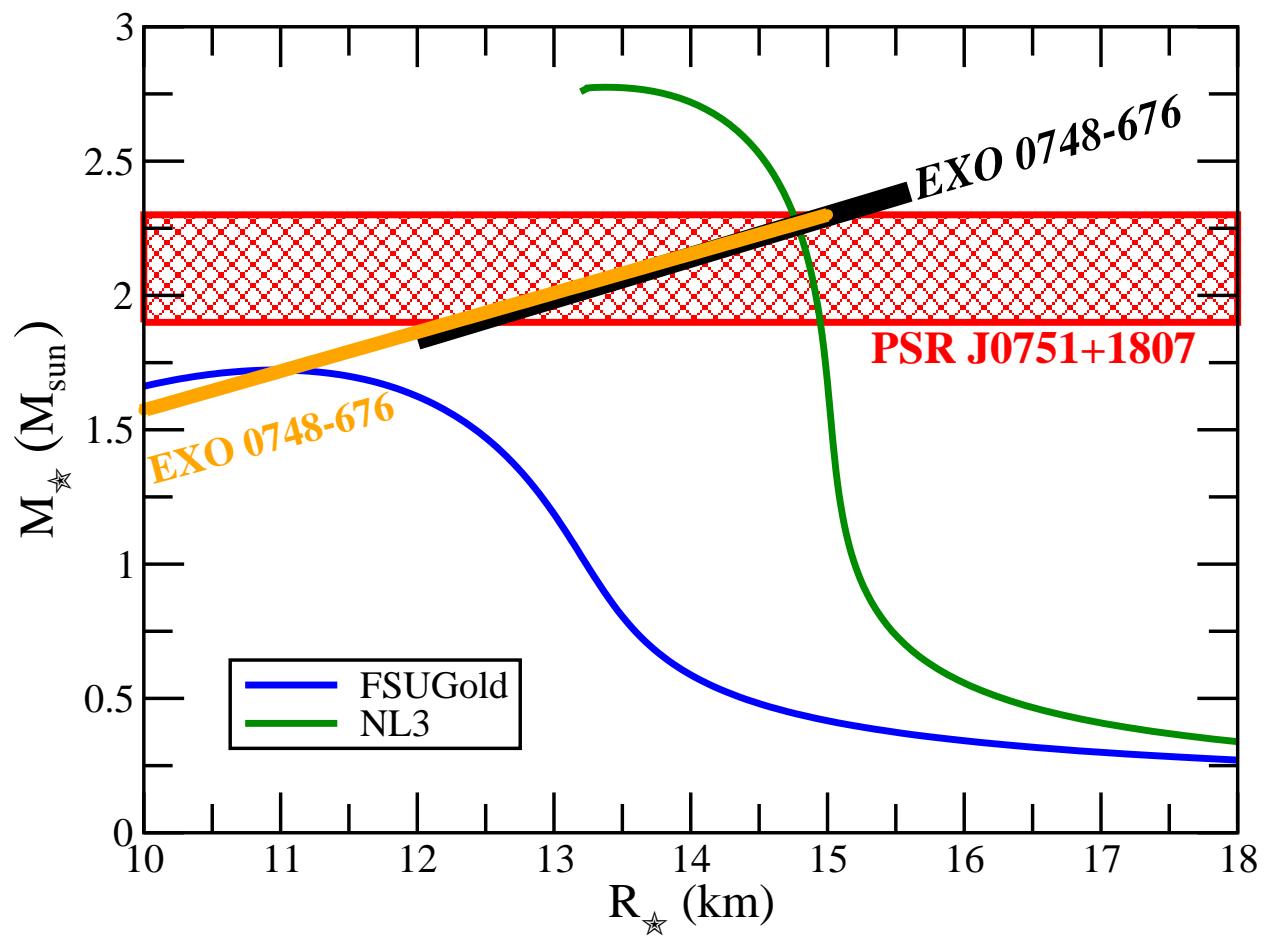

FIG. 5: (color online) Constraints on the mass-vs-radius relationship of neutron stars. Displayed in red is the allowed region as determined by the analysis of Nice and collaborators [17]. The black and orange solid lines result from the analyzes of EXO 0748-676 by Özel [21], and Villarreal and Strohmayer [44], respectively. Also shown are the theoretical predictions from the NL3 [1, 2] (green line) and FSUGold [5] (blue line) models.

The second observation that seems to suggest a hard equation of state is that of the low- 
mass X-ray binary EXO 0748-676. The first constraint on the equation of state from such an object came from the detection of gravitationally redshifted absorption lines in Oxygen and Iron by Cottam and collaborators [20]. By measuring a gravitational redshift of $z=0.35$, the mass-to-radius ratio of the neutron star gets fixed at $M / R \simeq 0.15$ (with $M$ expressed in solar masses and $R$ in kilometers). By incorporating additional constraints arising from Eddington and thermal fluxes, a recent analysis by Özel seems to place simultaneous limits on the mass and radius of the neutron star in EXO 0748-676. That is, $M \geq 2.10 \pm 0.28 M_{\odot}$ and $R \geq 13.8 \pm 1.80 \mathrm{~km}$ [21]. These limits are indicated by the black solid line in Fig. 5, An earlier determination of the spin frequency of the same neutron star by Villarreal and Strohmayer [44], when combined with the rotational broadening of surface spectral lines, yields an independent determination of the stellar radius of $R \approx 11.5_{-2.5}^{+3.5} \mathrm{~km}$. This estimate, when combined with the gravitational redshift, yields the orange line in Fig. 5. Note, however, that the use of rotational broadening to constrain the stellar radius has been put into question in Ref. [21]. Finally, mass-vs-radius predictions from the NL3 and FSUGold models are displayed in Fig. 5. The results clearly indicate the significantly harder character of the equation of state predicted by NL3 relative to FSUGold. This, even when both models predict practically identical properties for existent ground-state observables of finite nuclei. Based solely on these observations, NL3 with its stiff equation of state appears to fair far better than FSUGold, despite the fact that both breathing-mode energies and heavy-ion experiments seem to suggest that the NL3 equation of state is overly stiff.

\section{CONCLUSIONS}

Accurately calibrated relativistic models of nuclear structure have been enormously successful at describing a variety of ground-state properties throughout the periodic table by employing a relatively small number of effective parameters. Chief among these is the NL3 parameter set of Lalazissis, Ring, and collaborators [1, 2]. Yet under closer scrutiny, it was revealed, perhaps not surprisingly, that the properties employed in the calibration procedure of such models are insufficient to firmly pin down the equation of state even around saturation density. In an effort to lift this "degeneracy" the FSUGold model was conceived [5]. Relative to NL3, FSUGold includes two additional effective parameters. One of them [denoted by $\Lambda_{\mathrm{v}}$ in Eq. (11)] is responsible for a softening of the symmetry energy and should be firmly constrained by the pioneering Parity Radius Experiment at the Jefferson Laboratory that is scheduled to run in the early part of 2009 [32]. The other parameter [denoted by $\zeta$ in Eq. (11)] controls the high-density component of the equation of state and may be tuned to generate maximum neutron star masses that differ by almost one solar mass without significantly affecting the behavior of the equation of state near saturation density [25]. These two parameters - that are set to zero in the NL3 model - have a particularly dramatic impact on two observables: (a) the neutron-skin thickness of ${ }^{208} \mathrm{~Pb}$ and (b) the limiting mass of a neutron star. In both cases NL3 predicts significantly larger values than FSUGold, namely, $0.28 \mathrm{fm}$ vs $0.21 \mathrm{fm}$ for the former and $2.78 M_{\odot}$ vs $1.72 M_{\odot}$ for the latter. The main goal of this contribution was to test the validity of these models away from their region of applicability.

The validation of FSUGold - a model calibrated to various ground-state properties and collective excitations of finite nuclei — was implemented through a detailed comparison against recent theoretical, experimental, and observational constraints. These constraints emerged from the universal behavior of dilute Fermi gases with large scattering lengths [12], 
heavy-ion experiments that probe both the low- and high-density domain of the equation of state [13, 14, 15, 38], and astronomical observations that place limits on masses and radii of neutron stars [17, 21]. On the basis of these comparisons, it was concluded that FSUGold meets all the challenges, even when no attempt was ever made to incorporate these constraints into the calibration procedure. If at all, only the observational data seems to call FSUGold into question by suggesting a slightly harder equation of state. The promise of improved observations and analyzes with existent and future missions, such as Constellation $X$, offers the greatest hope for determining the high-density component of the equation of state.

The response to such future achievements should not be limited to an indiscriminate adjustment of parameters. After all, "knobs" can always be turned to reproduce a particular set of experiments and/or observations. Rather, models should aim at reproducing simultaneously a myriad of observables that probe the equation of state over a wide dynamic range. This should be one of the primary missions of all modern theoretical approaches. Only by adopting such strict standards one could test the validity and applicability of such models. Only then can one ensure the emergence of exotic phenomena.

\section{Acknowledgments}

The author is grateful to the Institute for Nuclear Theory at the University of Washington for its hospitality during the early phase of this work. The author is also grateful to Achim Schwenk for providing access to his theoretical results. This work was supported in part by the Department of Energy grant DE-FD05-92ER40750. 
[1] G. A. Lalazissis, J. Konig, and P. Ring, Phys. Rev. C55, 540 (1997), nucl-th/9607039.

[2] G. A. Lalazissis, S. Raman, and P. Ring, At. Data Nucl. Data Tables 71, 1 (1999).

[3] B. A. Brown, Phys. Rev. Lett. 85, 5296 (2000).

[4] R. J. Furnstahl, Nucl. Phys. A706, 85 (2002), nucl-th/0112085.

[5] B. G. Todd-Rutel and J. Piekarewicz, Phys. Rev. Lett 95, 122501 (2005), nucl-th/0504034.

[6] B. K. Agrawal, S. Shlomo, and V. K. Au, Phys. Rev. C72, 0143310 (2005), nucl-th/0505071.

[7] B. K. Agrawal, S. Shlomo, and V. K. Au, Phys. Rev. C68, 031304 (2003), nucl-th/0308042.

[8] G. Colo, N. Van Giai, J. Meyer, K. Bennaceur, and P. Bonche, Phys. Rev. C70, 024307 (2004), nucl-th/0403086.

[9] U. Garg et al., Nucl. Phys. A788, 36 (2007), nucl-ex/0608007.

[10] J. Carlson, S.-Y. Chang, V. R. Pandharipande, and K. E. Schmidt, Phys. Rev. Lett. 91, 050401 (2003).

[11] Y. Nishida and D. T. Son, Phys. Rev. Lett. 97, 050403 (2006), cond-mat/0604500.

[12] A. Schwenk and C. J. Pethick, Phys. Rev. Lett. 95, 160401 (2005), nucl-th/0506042.

[13] P. Danielewicz, R. Lacey, and W. G. Lynch, Science 298, 1592 (2002), nucl-th/0208016.

[14] M. B. Tsang et al., Phys. Rev. Lett. 86, 5023 (2001), nucl-ex/0103010.

[15] M. B. Tsang et al., Phys. Rev. C64, 054615 (2001).

[16] A. Ono, P. Danielewicz, W. A. Friedman, W. G. Lynch, and M. B. Tsang, Phys. Rev. C68, 051601 (2003), nucl-th/0305038.

[17] D. J. Nice et al., Astrophys. J. 634, 1242 (2005), astro-ph/0508050.

[18] J. M. Lattimer and M. Prakash, Phys. Rept. 442, 109 (2007), astro-ph/0612440.

[19] J. M. Lattimer and M. Prakash, Science 304, 536 (2004), astro-ph/0405262.

[20] J. Cottam, F. Paerels, and M. Mendez, Nature 420, 51 (2002), astro-ph/0211126.

[21] F. Ozel, Nature 441, 1115 (2006).

[22] B. D. Serot and J. D. Walecka, Adv. Nucl. Phys. 16, 1 (1986).

[23] B. D. Serot and J. D. Walecka, Int. J. Mod. Phys. E6, 515 (1997), nucl-th/9701058.

[24] J. D. Walecka, Annals Phys. 83, 491 (1974).

[25] H. Mueller and B. D. Serot, Nucl. Phys. A606, 508 (1996), nucl-th/9603037.

[26] C. J. Horowitz and J. Piekarewicz, Phys. Rev. Lett. 86, 5647 (2001), astro-ph/0010227.

[27] C. J. Horowitz and B. D. Serot, Nucl. Phys. A368, 503 (1981).

[28] D. H. Youngblood, C. M. Rozsa, J. M. Moss, D. R. Brown, and J. D. Bronson, Phys. Rev. Lett. 39, 1188 (1977).

[29] J. Boguta and A. R. Bodmer, Nucl. Phys. A292, 413 (1977).

[30] C. J. Horowitz and J. Piekarewicz, Phys. Rev. C64, 062802 (2001), nucl-th/0108036.

[31] C. J. Horowitz, S. J. Pollock, P. A. Souder, and R. Michaels, Phys. Rev. C63, 025501 (2001), nucl-th/9912038.

[32] R. Michaels, P. A. Souder, and G. M. Urciuoli (2005), URL http://hallaweb.jlab.org/parity/prex.

[33] G. A. Baker, Phys. Rev. C60, 054311 (1999).

[34] H. Heiselberg, Phys. Rev. A63, 043606 (2002), cond-mat/0002056.

[35] B. Friedman and V. R. Pandharipande, Nucl. Phys. A361, 502 (1981).

[36] T. X. Liu et al., Phys. Rev. C69, 014603 (2004), nucl-ex/0210004.

[37] B. G. Todd and J. Piekarewicz, Phys. Rev. C67, 044317 (2003), nucl-th/0301092. 
[38] D. V. Shetty, S. J. Yennello, and G. A. Souliotis, Phys. Rev. C75, 034602 (2007), nuclex/0505011.

[39] A. Ono, P. Danielewicz, W. A. Friedman, W. G. Lynch, and M. B. Tsang (2005), nuclex/0507018.

[40] D. V. Shetty et al. (2006), nucl-ex/0603016.

[41] B.-A. Li and L.-W. Chen, Phys. Rev. C72, 064611 (2005), nucl-th/0508024.

[42] I. Sagert, M. Wietoska, J. Schaffner-Bielich, and C. Sturm (2007), arXiv:0708.2810 [astro-ph].

[43] D. Blaschke, T. Klahn, and F. Sandin (2007), arXiv:0708.4216 [nucl-th].

[44] A. R. Villarreal and T. E. Strohmayer, Astrophys. J. 614, L121 (2004), astro-ph/0409384. 